\title{
Masculinidades alternativas: un modelo para alcanzar la transformación desde la educación social
}

\author{
Alternative masculinities: A model to achieve \\ transformation from social education
}

\author{
Patricia Cardeńosa Iglesias ${ }^{a}$ ORCID: 0000-0001-6908-7966 \\ Leire Darretxe Urrutxi'b ORCID: 0000-0001-7468-7915 \\ Nekane Beloki Arizti\} ${ }^{c}$ ORCID: 0000-0002-2728-845X
}

Recibido: $15 / 04 / 2020 \bullet$ Aprobado: $17 / 07 / 2020$

Cómo citar: Cardeñosa Iglesias, P., Darretxe Urrutxi, L., \& Beloki Arizti, N. (2021). Masculinidades alternativas: un modelo para alcanzar la transformación desde la educación social. Ciencia y Educación, 5(1), 147-158. https://doi.org/10.22206/cyed.2021.v5i1. pp147-158

\section{Resumen}

El presente artículo propone unas orientaciones para incorporar el Feminismo y las "masculinidades alternativas" en la acción socioeducativa, llevada a cabo por educadores y educadoras sociales en sus diferentes ámbitos de trabajo. A partir de una revisión teórica sobre el concepto de masculinidades alternativas y su importancia para la transformación de las sociedades, este enfoque de género pone al hombre como principal protagonista, en el camino hacia la igualdad. A través de estas líneas reflexivas plantearemos cómo y de qué manera, el modelo de masculinidad hegemónica construido desde el patriarcado crea una diferencia desigualitaria en función del sexo, posicionando a la mujer en una situación de desventaja permanente y ejerciendo consecuencias negativas también para muchos hombres. Ante esto, enfocaremos las masculinidades alternativas como cuestionadoras de ese modelo, ya que su enfoque crítico, y por lo tanto sus propuestas, son indispensables en toda acción encaminada a la transformación social.

Palabras clave: masculinidades alternativas; igualdad de género; masculinidad hegemónica; educación social; feminismo.

\begin{abstract}
This paper presents a series of guidelines to incorporate Feminism and alternative masculinities in the socio-educational action. A theoretical reflection of the concept of "alternative masculinities" has been developed. The gendered-based approach that has been implemented puts the male as the main actor in the advancement towards gender equality. Throughout this paper we describe how and in which way the hegemonic masculinity model, constructed from the patriarchy, exerts an unfavourable treatment depending on the gender. Thus, placing women at a permanent disadvantage and exerting negative consequences also for many men. We focus the alternative masculinities as the main drivers questioning that model due to the fact that its critical approach and its ideas are indispensable in any action towards social transformation. Similarly, we provide orientation for the educational practice of social educators from the perspective of alternative masculinities.
\end{abstract}

Keywords: alternative masculinities; gender equality; hegemonic masculinity; educational practice; Feminism.

\footnotetext{
a Izan Intervención Social y Comunitaria SL, España. Correo-e: patricarde10@gmail.com

b Universidad del País Vasco/Euskal Herriko Unibertsitatea (UPV/EHU), España. Correo-e: leire.darretxe@ehu.euss

c Universidad del País Vasco/Euskal Herriko Unibertsitatea (UPV/EHU), España. Correo-e: nekane.beloki@ehu.eus
} 


\section{Introducción}

El concepto de patriarcado surge en el año 1970, a partir de la publicación del libro de Kate Millet, titulado Política Sexual (1969). Desde entonces, este término es considerado como un instrumento muy útil para orientar y cambiar la situación en el mundo de muchas mujeres. Así Fontela (2020) define el patriarcado como un sistema de relaciones sociales creado por los varones oprimiendo a las mujeres, tanto por medios pacíficos como violentos.

$\mathrm{Al}$ igual que los hombres, las mujeres son y siempre han sido actoras y agentes en la historia. Nunca han estado al margen, por el contrario, han tenido mucho que ver en la formación y el desarrollo de la sociedad (Lerner, 1990). Asimismo, sabemos que representan la mitad de la humanidad, y en algunos casos, más de la mitad (Banco Mundial, 2020); por ello, han compartido con los hombres el mundo y el trabajo.

Hasta un pasado reciente, la mayoría de los historiadores han sido hombres que han registrado lo que su mismo género ha hecho, experimentado y considerado que era importante. Así lo indica Radkau (1986), quien afirma que poseemos la percepción de una historia "general", de la que las mujeres son una parte "especial", pero realmente es historia masculina contada por hombres. Todo ello, nos puede llevar a la conclusión de que las vivencias y experiencias de las mujeres no han sido registradas y, en muchos casos, no se ha tenido en cuenta su interpretación. Esto nos acerca a la idea de la existencia de un dominio patriarcal muy arraigado con raíces históricas muy profundas.

\section{La construcción del género masculino}

Junto a muchas aportaciones feministas: Amorós, 2006; De Beauvoir, 1981; Carabí, 2000; Castro y Spoturno, 2020; Facio y Fries, 1999; Fontenla, 2020; Freedman, 2004; Lerner, 1990; Medina-Vicent, 2020; Millet, 1995; Radkau, 1986; Reverter y Medina-Vicent, 2020; Torras-Gómez et al., 2020; Varela, 2020); así como diversas investigaciones, entre ellas, Aresti (2020); Azpiazu (2017); Bonino (2002); Connell (1987); Kimmel (2001); existe una vasta producción sobre la construcción de género masculino, es decir, la formación de la masculinidad.

Así, Bergara et al. (2008) explican que la construcción de género es uno de los primeros y más importantes aprendizajes que nos enseñan la familia, la escuela, la religión y la sociedad en la que crecemos. Es decir, que el propio proceso de socialización en el que se desarrollan niñas y niños, se ocupa de impulsar las conductas consideradas correctas para cada sexo y de contener las que no se adaptan a las actitudes y roles esperados, ayudando así a preservar los sistemas patriarcales (Kaufman, 1994). Por ejemplo, Bonino (2002) indica que varios autores como Connell (1987) o Kimmel (2001) "han estudiado la constitución de diferentes manifestaciones de la masculinidad en contextos y momentos históricos diferentes, llamando a esas manifestaciones «masculinidades», aludiendo a su importancia en tanto diversidades de un único modelo predominante de masculinidad" (p. 7). Este modelo al que se refiere el autor es la masculinidad tradicional o con mayor precisión la masculinidad hegemónica.

Una definición acertada de la masculinidad hegemónica es la que señala Espada (2004) como una serie de doctrinas que privilegian a algunos hombres al vincularlos con ciertas formas de dominio. Dicho autor, basándose en una investigación de Carrigan, Connell y Lee en 1985, explica que la masculinidad hegemónica configura formas victoriosas de "ser hombre", y como consecuencia, marcan otras expresiones masculinas como inadecuadas o inferiores. Por su parte, Bonino (2002) define la masculinidad hegemónica como:

La configuración normativizante de prácticas sociales para los varones predominante en nuestra cultura patriarcal, con variaciones pero persistente. (...) Relacionada con la voluntad de dominio y control, es un corpus construido sociohistóricamente, de producción ideológica, resultante de los procesos de organización social de las relaciones mujer/hombre a partir de la cultura de dominación y jerarquización masculina. (p. 9)

Por otro lado, la virilidad se construye socialmente (Kimmel, 1997), por tanto, comprendemos que este modelo de masculinidad hegemónica responderá al resultado de una sociedad patriarcal que 
buscará una argumentación sistémica de diversas normas sexuales y de género, establecidas socialmente, para manifestar y reafirmar el poder del hombre sobre la mujer (Kaufman, 1994).

Ahora bien, después de observar y deliberar en torno a la construcción de la masculinidad hegemónica y siguiendo a Azpiazu (2017), podemos hablar de colectivos de hombres que reconociendo la masculinidad tradicional buscan una transformación de su propia masculinidad hacia patrones menos dañinos y más igualitarios, donde se construye la igualdad de género desde los hombres, transformando la masculinidad hacia nuevas maneras de ser hombre. Así, la igualdad de género implica la necesidad de un orden social donde mujeres y hombres puedan desarrollar sus potencialidades como seres humanos (Facio y Fries, 1999). En definitiva, entendemos que se trata de conseguir un cambio en aquellas formas de vida e ideologías que legitiman la desventaja femenina, la cual se crea cuando se entiende la inferioridad de la mujer como algo natural.

Ante este panorama, Ibarra (2005) identifica el

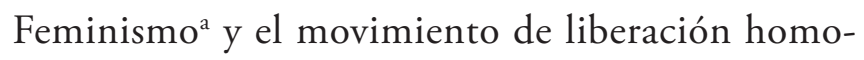
sexual como antecedentes de las masculinidades alternativas. Por un lado, el feminismo, como movimiento de transformación social, procura dar respuesta a la misma cuestión que pretenden abordar las masculinidades alternativas; acabar con los estereotipos y las desigualdades que limitan las libertades de las mujeres, pero también de algunos hombres; desigualdades que surgen de la construcción social de la masculinidad hegemónica. Por otro lado, aludiendo al movimiento de liberación homosexual, este se centra en la afirmación de distintas identidades de género alternativas a la masculinidad hegemónica. Como podemos observar, ambos movimientos precursores de las masculinidades alternativas repercuten en el inicio de un desequilibrio de la masculinidad hegemónica. Al entrar en crisis el modelo dominante de masculinidad, comienzan a surgir nuevos planteamientos en torno a ella.
Carabí (2000) afirma, que el origen de un nuevo movimiento de hombres que cuestiona esta masculinidad hegemónica también nace de aquellos que en los ańos 70 participaban en las tareas del hogar y en la crianza de sus hijos e hijas. Este cuestionamiento de la masculinidad ha ocasionado el que hoy en día, en nuestro medio, se haya experimentado un creciente interés en el tema de las masculinidades alternativas, como un movimiento con un factor transformador de género, como modelo preventivo ante la violencia de género (Duque, 2015; Flecha et al., 2013) que busca la igualdad, el respeto y la diversidad. No obstante, en un pasado, las mujeres eran el único objeto de estudio en las investigaciones de género; solo cuando se empezó a cuestionar el modelo de masculinidad dominante, a partir de los años 80 , se incluyó la masculinidad bajo el nombre de los Men's studies o estudios de género en masculino, estudios desarrollados principalmente en los países anglosajones (Jociles, 2001).

Por ejemplo, Bonino (2002) hace referencia en sus estudios al cuestionamiento del modelo hegemónico masculino. Entre sus distintas publicaciones, este autor trata de mostrar cuál es el verdadero camino para alcanzar una auténtica igualdad de género a escala global, a través de las masculinidades alternativas. En la misma línea, Carabí (2000), quien también señala la posible crisis de la masculinidad hegemónica, explicará que la evolución del hombre es crucial para conseguir el cambio, y que debe tomar conciencia de sus privilegios. Esta es la única forma de entender de qué factores está compuesto el modelo hegemónico de masculinidad, y qué barreras interfieren en dicha construcción. En esta línea, Soto (2013) nos ofrece la siguiente definición de estas masculinidades alternativas.

El concepto de nuevas masculinidades se puede entender como un movimiento incipiente de hombres o grupos de hombres que abogan por la igualdad entre hombres y mujeres en todos los aspectos de la vida y que luchan por erradicar la violencia física o psicológica a mujeres, niños, ancianos y hombres. Del mismo modo,

a. En este artículo, al igual que hace Alda Facio, mencionada por De las Heras, 2009, p. 46, utilizaremos el término "Feminismo con "F" mayúscula" para designar al conjunto de movimientos feministas. 
las nuevas masculinidades promueven la crítica a la masculinidad hegemónica a quienes identifican como la responsable de la negación de los derechos de mujeres y hombres, así como la reguladora del mantenimiento de los propios privilegios en desmedro de lo que ella define como normal o patológica. (p. 95)

\section{Consecuencias positivas de las masculinidades alternativas}

Como venimos explicando, "los hombres organizados alrededor de la idea de la igualdad se centran desde hace más de 20 años en las estrategias de crecimiento personal" (Bergara et al., 2008, p. 51). Aunque en los últimos tiempos han surgido iniciativas tanto en el estado espańol como a nivel internacional (AHIGE, RHXI, Gizon Sarea, CORIAC, Gizonduz, etc.) de grupos de hombres por la igualdad, con un carácter más público o social.

Entendemos que estos grupos reconocen la responsabilidad masculina en el mantenimiento de la desigualdad con las mujeres y ejercen una autocrítica sobre el propio ejercicio del poder. Los mismos, promueven practicar la igualdad con las mujeres, el activismo social, la formación de grupos de reflexión de hombres, etc., para desmontar el ideal de la masculinidad hegemónica y construir modelos que contribuyan a prevenir y a erradicar la desigualdad de género. Siguiendo esta línea, Boscán (2008) asegura que la masculinidad debe constituirse desde distintas opiniones, tanto masculinas como femeninas, además de plantearse como una concepción "abierta, plural, flexible y dinámica” que permita integrar los diferentes modos de expresar la masculinidad.

Por consiguiente, lo grupos de nuevos hombres tienen como tarea el análisis y la deconstrucción del impacto negativo que tiene para los varones la masculinidad hegemónica. Estos grupos se organizan y actúan para deconstruir la masculinidad sexista (Bergara et al., 2008), explicando toda una serie de propuestas que abordan la teoría de estos colectivos de varones. Entre ellas cabe destacar que:
... rechazan el modelo masculino dominante, el sometimiento acrítico al corporativismo viril y la homofobia, no se avergüenzan de la influencia de las mujeres en sus vidas y proponen el activismo social, la investigación académica y la formación de grupos de reflexión de varones para deconstruir el ideal de masculinidad tradicional y construir masculinidades alternativas, romper la complicidad masculina antisexista y practicar la igualdad con las mujeres. (Bonino, 2003, p. 117)

En efecto, el modelo de masculinidad alternativa supone una nueva opción a las estructuras de la masculinidad hegemónica, para así erigir relaciones basadas en la igualdad, luchar contra la violencia de género y mostrar a los hombres que desempeñan este modelo interesante (Flecha et al., 2013).

Para dar más fuerza y sustento a este ideal, según la Asociación de Hombres por la Igualdad de Género (AHIGE), dicho modelo: colabora en la construcción de personalidades más justas, solidarias y no discriminatorias, aporta a la superación del machismo creando modelos masculinos alternativos al tradicional, genera mayor capacidad para gestionar las emociones, la autoestima y la seguridad en uno/a mismo/a, contribuye a una sexualidad más completa y satisfactoria, así como a una paternidad más cercana, responsable y solidaria, generando también formas nuevas de relacionarse en pareja y con otros hombres (AHIGE, 2020).

Ahora bien, podemos apreciar que, pese a su relevancia y esencialidad, el modelo de masculinidades alternativas es, con diferencia, uno de los que menos miembros de adeptos reúnen. Esto nos hace cuestionarnos si verdaderamente esta crisis de masculinidad está avanzando sin detenerse o, por el contrario, forma parte de un desarrollo pausado y con varios elementos para tener en cuenta en su evolución.

\section{Consecuencias negativas de la masculinidad hegemónica}

Como hemos aclarado anteriormente, el poder y la masculinidad hegemónica están estrechamente relacionados (Connell, 1987; Kaufman, 1994). “El 
poder asociado a la masculinidad exige poseer algunas características, tales como ganar, mandar, lograr objetivos y ser fuerte y duro. Estas características genéricas atribuidas al hombre, le otorgan un dominio sobre la mujer" (Hardy y Jiménez, 2001, p. 80). Así, de acuerdo con Bourdieu (2000), el varón demuestra su virilidad siendo el más fuerte, dominante y sabio. El poder también representa controlar sentimientos, emociones y necesidades afectivas, para no perder el dominio ni el control sobre las demás personas, y para contrarrestar el miedo a que se le atribuyan características femeninas, que están mal vistas en la sociedad (Kaufman, 1994).

Observamos entonces, que la masculinidad hegemónica asigna a los individuos una forma estereotipada y estricta de ser, razonar e intervenir en la sociedad. Como indica Bourdieu (2000) "los hombres también están prisioneros y son víctimas subrepticias de la representación dominante” (p. 67). Definitivamente, este modelo de hombre tradicional con sus privilegios y poderes supone graves consecuencias tanto para las mujeres como para los hombres.

En primer lugar y a modo de síntesis, veamos algunos de los malestares y problemáticas que suscitan esta identidad (hegemónica) a los propios hombres. El hombre representa la imagen de suministrador económico principal de la familia, lo que para él supone una gran presión, ya que le toca encargarse de muchas cuestiones relacionadas con la vida pública (Bonino, 2000). Además, esta situación hace que el varón, aun deseándolo, no pueda disfrutar de aspectos, como reforzar el vínculo familiar y todas las ventajas afectivas que ello conlleva.

Según Hardy y Jiménez (2001), la violencia y el enfrentamiento son los modos a través de los cuales el hombre se hace respetar, tanto por parte de las mujeres como por parte de los hombres, estando vinculado al riesgo. "El hombre necesita que su vida se marque por hechos heroicos y, al mismo tiempo, tener historias para contar" (Fagundes, 1995; Gastaldo, 1995, citados en Hardy y Jiménez, 2001, p. 84). Sabemos que en nuestra sociedad los hombres tienen una menor expectativa de vida que las mujeres, y ello puede ser debido a una mayor exposición a riesgos y peligros, como parte del rol de ser hombre, como, por ejemplo, exposición a la violencia y a las adicciones. Así, en el ámbito laboral, los trabajos a los que acceden los hombres tienen mayores índices de peligrosidad y exigen una mayor capacidad física. Es decir, existe una división social del trabajo relacionada con el género (Hardy y Jiménez, 2001).

Por otro lado, según Otegui (1999), la representación hegemónica de la masculinidad se configura principalmente en torno a la genitalidad, de modo que "el aparato genital masculino se instituye como el resumen, ícono y sustancia de la masculinidad” (p. 154). Otro estudio sobre las problemáticas de la masculinidad tradicional muestra, que cuando el ser humano intenta desprenderse de su capacidad emotiva, tiende a deshumanizarse (Lorente, 2009).

Por otro lado, la desventaja de la mujer frente al varón todavía está presente en la mayoría de sectores vitales. Por ejemplo, como señala Emakunde (2018), se visibiliza una situación de desventaja en las mujeres frente a los hombres en el ámbito laboral, el ámbito de los cuidados, entre otros.

Resumiendo, la existencia de constructos sociales de género que abogan por un comportamiento ya establecido entre ambos sexos, será lo que provoque situaciones de desigualdad tanto en mujeres como en aquellos varones que no actúen conforme a la norma establecida. Por todo esto, desde las masculinidades alternativas, se intentará suprimir el modelo de masculinidad tradicional como ideal a seguir, y se procurará normalizar y fomentar nuevos modelos y maneras de ser hombre.

\section{Educación social, género y masculinidades alternativas}

En los últimos años, contamos con varias publicaciones (Duque, 2015; Flecha et al., 2013; Leiva et al., 2015; Peña y Ríos, 2011; Portell y Pulido, 2012; Ríos, 2015) que relacionan directamente el ámbito educativo y socioeducativo con la perspectiva de género desde los estudios de masculinidades. Algunas 
muestran que las nuevas masculinidades suponen una contribución clave para la superación de la violencia contra las mujeres. Entre algunas de estas investigaciones, existe una idea muy interesante (Peńa y Ríos, 2011; Ríos, 2015; Rodríguez, 2017;) que las relaciona con la educación liberadora de Freire (1978), la comunicación y el diálogo.

A pesar de estos avances importantes, cabe destacar que en observatorios y diversas asociaciones profesionales de mujeres, la educación formal y la no formal generalmente están poco representadas (Ytarte, 2015).

En cuanto a la presencia de esta mirada, Leiva et al. (2015) argumentan que el Feminismo es un tema secundario en el campo de la Educación Social. Ytarte (2015), asegura que en páginas web que representan la educación social, el género no se muestra expresamente reconocido a diferencia de otros contenidos como la justicia social, la igualdad de oportunidades, la exclusión social, el Estado de Derecho, la sociedad del bienestar, etc. Si analizamos el código deontológico de los y las educadoras sociales, podemos comprobar en el capítulo tres, el cual consta de 32 artículos que, aunque todos cuentan con un lenguaje inclusivo, ninguno de ellos contiene el concepto de igualdad de género, ni la palabra género, ni relaciona la profesión con el Feminismo. Sin embargo, recientemente ha aparecido una publicación que aborda dicho código deontológico desde la práctica profesional y que sí tiene en cuenta la perspectiva de género (Pantoja et al., 2018), en el cual está más presente la igualdad de género.

Debido a que en el colectivo de profesionales de la educación social predomina el género femenino, y a que nuestro Colegio profesional del País Vasco se denomina Colegio de Educadoras y Educadores Sociales, se ha tomado la decisión de colocar en primer lugar el género femenino en este capítulo. (Pantoja, 2018, p. 107)

Aludiendo a la importancia de esta mirada, aunque cada vez somos más conscientes de la necesidad de trabajar el tema de la igualdad de género en la educación formal y no formal (Bas, 2015; Leiva et al., 2015; Llano et al., 2020; Ríos, 2015; Ytarte, 2015) y se creen avances poco a poco, es conveniente conocer la importancia de este enfoque como tema principal en una profesión como la educación social. Esta disciplina debe convertir en objetivo la igualdad de género y combatir frente a la situación de discriminación y violencia que sufren las mujeres. Los espacios socioeducativos, pueden ser espacios de diálogo e interacción, y estos son los idóneos para reflexionar en torno a los procesos de socialización basados en la masculinidad hegemónica que tenemos tanto los hombres como las mujeres (Flecha et al., 2013).

Podemos afirmar entonces, que la educación y la formación de profesionales es un requisito imprescindible para el desarrollo de las sociedades y que, como seńalan algunos autores y autoras (Azpiazu, 2017; Bonino, 2000, 2003; Boscan, 2008; Carabí, 2000; Connell, 1987; Kimmel, 2001; Martín y Vila, 2015; Marugán, 2020), hablar de la igualdad de género es una cuestión social, de todos los sexos, ya que el origen y los efectos de este constructo cultural son el reflejo de los valores hegemónicos en los que se nos enseña únicamente cómo ser hombre y mujer, olvidando otros modelos menos dañinos para los distintos géneros. Con las masculinidades alternativas, la configuración de la hombría y de las relaciones sociales entre géneros es más equitativa, respetuosa, crítica y transformadora (Azpiazu, 2017; Bonino, 2003; Soto, 2013). La concesión de legitimidad del modelo de masculinidades alternativas no solo demostraría su veracidad y autenticidad, sino que supondría el reconocimiento de la capacidad y derecho que tendría cualquier persona para el ejercicio de este.

\section{Propuesta de incorporación de la perspectiva de masculinidades alternativas en la acción socioe- ducativa}

Como profesionales del campo de la educación social, debemos expresar, diversas maneras de pedagogía reflexiva y formar maniobras didácticas con las que podamos abordar la construcción de identidades masculinas alternativas (Vila y Martín, 2015). Para ello, la percepción que tengamos las y los profesionales de la 
educación, sobre la construcción del género es determinante para afrontar y contribuir hacia la equidad entre mujeres y hombres (Rodríguez, 2017).

Por todo esto, como educadoras y educadores sociales, nos vemos en la disposición de plantear algunas orientaciones a nivel político, institucional y educativo que permitirían un mayor avance en el plano de la educación social, para la transformación de la ciudadanía en clave de igualdad de género y justicia social.

A. ugerimos la utilización de un lenguaje inclusivo, lo que supone retirar el único uso del masculino singular o plural como representación de ambos sexos, sustituyéndolo por otras expresiones más democráticas y representativas o por la incorporación también del femenino singular o plural. Es necesario el uso del lenguaje inclusivo, porque inconscientemente transmitimos androcentrismo y omitimos lo femenino (Martín y Vila, 2015). Martín y Vila (2015) nos recuerdan que es tarea de las y los educadores "revisar el lenguaje utilizado, que evite los fenómenos sexistas de carácter léxico" (p. 147), ya que las palabras representan las ideas que se llevan a cabo mediante los comportamientos (Peña y Ríos, 2011). Y no solo eso, de acuerdo con Freire (1997) "siempre digo hombres y mujeres porque decir solamente hombres es inmoral (...) esto que parece una cuestión de gramática obviamente no lo es" (p. 24).

A. Queremos destacar la importancia que tiene en el modelo de masculinidades alternativas en la paternidad activa responsable. Debemos educar y guiar hacia un proceso profundo de concienciación, para que los hombres se involucren en la conciliación con corresponsabilidad social. Para ello, también es necesaria la implementación de políticas públicas paritarias, ya que, sin la presencia de los hombres, el camino hacia la igualdad de género será más lento (Aguayo y Kimelman, 2016).

B. La Plataforma por Permisos Iguales e Intransferibles de Nacimiento y Adopción propone una reforma legislativa, que se basa en la igualdad de los permisos de nacimiento y/o adopciones intransferibles, de igual duración, con la misma parte obligatoria y pagada al $100 \%$. Además, el cuidado y la responsabilidad hacia las demás personas puede aumentar la autoestima, fomentar el desarrollo personal y favorecer las relaciones sociales (Bergara et al., 2008).

C. Otra línea de acción educativa consistiría en eliminar de nuestras intervenciones socioeducativas, tanto los estereotipos como la asignación de roles en función del sexo. La estrategia de igualdad de género 2018-2023, aprobada en mayo del 2018 por el Comité de Ministros y Ministras del Consejo de Europa, tiene entre dos de sus seis áreas prioritarias: Prevenir y combatir los estereotipos de género y el sexismo; y Lograr la incorporación de la perspectiva de género en todas las politicas y medidas en diferentes ámbitos como la educación, entre otros. A través de espacios comunicativos, donde se genere diálogo y reflexión sobre roles o comportamientos hegemónicos, estamos incidiendo en el proceso de socialización (Peña y Ríos, 2011). Como hemos dicho anteriormente, contamos con aportaciones cuya base pone el foco central en la teoría dialógica y liberadora de Freire (1978). Esta teoría de la pedagogía crítica, planteada desde las masculinidades alternativas fomenta nuevas formas de ser hombre rompiendo con la atribución de roles y estereotipos en entornos educativos, ya que genera espacios para el diálogo que posibiliten reflexionar sobre esta perspectiva (Ríos, 2015). Si las interacciones entre las personas son dialógicas reproducirán relaciones basadas en la igualdad, el consenso y con el poder de transformar (Peña y Ríos, 2011). Si se permite el diálogo social se harán más visibles y se considerarán nuevos referentes masculinos (Ríos, 2015).

D. Proponemos la utilización de buenas prácticas de Tecnologías de la Información y Comunicación (TIC), relacionadas con el Feminismo. En España y Europa existen varios recursos 
significativos tanto para el área personal como social que nos pueden ayudar en esta labor: Alfa Beta, Conectad@s, Asociacionismo/Redes, Amedna-neeze, El portal de la conciliación, Convive, Servicio del IAM especializado en violencia de género, European Center For Women and Technology, European Professional Women's Network; y muchos más relacionados con los siguientes campos: creación y fortalecimiento empresarial, desarrollo profesional y empleo, e-igualdad, estudios y análisis, mainstreaming de género, etc. Estos recursos ofrecen a la Educación Social la posibilidad de construir y proporcionar acceso a espacios igualitarios, que permitan a las personas intercambiar pensamientos, experiencias, sentimientos, valores, así como acceso a formación e información actualizada permanentemente. Además, es una buena herramienta para difundir los valores universales (Belloch, 2020).

E. Sugerimos la incorporación del modelo de masculinidades alternativas al aprendizaje y a la formación de las educadoras y los educadores sociales. Bas (2015) afirma que la formación está ligada a la preparación de profesionales y, por tanto, es una parte importante para el desarrollo de la profesionalización. En el caso del grado, en educación social el currículum educativo integra el género de modo transversal; no obstante, creemos necesarias mayor número de acciones formativas concretas que impliquen una sensibilización, aprendizaje o concienciación en materia de masculinidades alternativas. Además, sabemos que la limitación de la transversalidad de género en las titulaciones es persistente, siendo un tanto curioso que, en la oferta de máster, grupos de investigación y redes que trabajan el tema de la igualdad de género, la participación de las facultades de Educación Social apenas sea visible (Ytarte, 2015). Pensamos que es necesaria una formación que incorpore tanto el feminismo como la perspectiva de las masculinidades alternativas, como opción a una visión del mundo centrada en el varón, que obvia e invisibiliza a las mujeres, visto que favorece la prevención de la violencia machista (Duque, 2015) y otorga estrategias para una praxis socioeducativa en materia de género.

B. Como parte de la comunidad educativa, los educadores y educadoras deberíamos realizar y profundizar en estudios e investigaciones acerca de diversos temas que formen parte del enfoque de género, como son las masculinidades alternativas. También puede ser parte de nuestra profesión presentar estos estudios e investigaciones a personas con responsabilidades públicas: cargos técnicos en igualdad, cargos en ayuntamientos, diputaciones, escuelas públicas, etc. Asimismo, formaría parte de nuestra labor el posibilitar y potenciar actos comunicativos en encuentros, charlas, formaciones etc. (Soler y Flecha, 2010), en diversas instituciones privadas de ámbito social y de educación formal y no formal: asociaciones, universidades, centros comunitarios, residencias, centros educativos, etc. El 20 de noviembre del 2019 se organizaron las primeras jornadas sobre Perspectiva Feminista en la Intervención Socioeducativa en la Universidad de Deusto, organizadas por la Facultad de Psicología y Educación, de la mano del Colegio de Educadoras y Educadores Sociales del País Vasco, donde se analizó y reflexionó en torno a la incorporación del Feminismo en el campo de la Intervención Social y Educativa. Entre muchas de las temáticas tratadas estuvieron presentes las masculinidades alternativas.

C. Resulta adecuado saber que la mayoría de órganos representativos de la Educación Social, está ocupada por hombres, especialmente los cargos más altos, como presidencia o vicepresidencias. Sin embrago en los cargos secundarios como las tesorerías o las secretaría, ocurre lo contrario, la proporción se invierte (Ytarte, 2015). Por ello es necesario que haya una igualdad de género real en relación con poder dentro las instituciones y órganos representativos de la Educación Social. Para que esto 
sea posible, sería adecuado analizar los procesos y estrategias de participación, así como el proceso de toma de decisiones de los órganos representativos de la educación social, de las personas colegiadas y de estudiantes del grado de Educación Social, a través de varios recursos que permiten detectar y gestionar necesidades de participación (González, 2019), como pueden ser consejos consultivos, servicios de información orientación y asesoramiento, planes estratégicos, etc.

\section{Conclusiones}

A través del análisis que hemos realizado, se evidencia que vivimos en un contexto patriarcal en el que predomina la masculinidad hegemónica, lo cual repercute en la subordinación que vienen sufriendo las mujeres en el día a día, a lo largo de la historia y en el presente. En consecuencia, las masculinidades alternativas nacen del Feminismo y del movimiento de liberación homosexual. Este modelo, proporciona otra forma de vivir la hombría más libre, igualitaria, respetuosa, no violenta, transformadora y que actúa como método preventivo ante la violencia de género.

Así, la desigualdad sufrida por la mujer durante siglos lleva a la sociedad a desear, exigir y respaldar que muchos varones se inclinen por conductas, posturas y prácticas igualitarias. Esto tiene que ver con el hecho de que la igualdad es un derecho humano. Tanto así que, se ha conseguido que en la Declaración Universal de Derechos Humanos se eliminaran las referencias a "los hombres", sustituyéndolo por un lenguaje inclusivo, como "todos los seres humanos", "todas las personas", "todo individuo", etc.

En el ámbito de la educación social nuestra lucha para favorecer sociedades más justas debe ser política y ética. No obstante, la utilización de un lenguaje no sexista en los escritos no es suficiente (Ytarte, 2015). La educación social necesita de referencias que garanticen un vigoroso enfoque de género en su organización, en sus políticas, estrategias, intervenciones, programas y proyectos. Por consiguiente, a través de este artículo hemos compartido algunas orientaciones que pueden contribuir a la justicia social apostando por estas masculinidades alternativas, puesto que este enfoque resulta esencial e indispensable para una educación social crítica, equitativa y transformadora para una sociedad democrática.

Con todo lo argumentado, finalmente, aportamos un decálogo a modo de material didáctico (figura 1) que contiene diez principios basados en las siete orientaciones planteadas anteriormente, con las principales acciones que podemos seguir para emplear el Feminismo y la perspectiva de las masculinidades alternativas en el ámbito de la educación social.

\section{Figura 1}

Decálogo de orientaciones para educadoras y educadores sociales desde la perspectiva de masculinidades alternativas

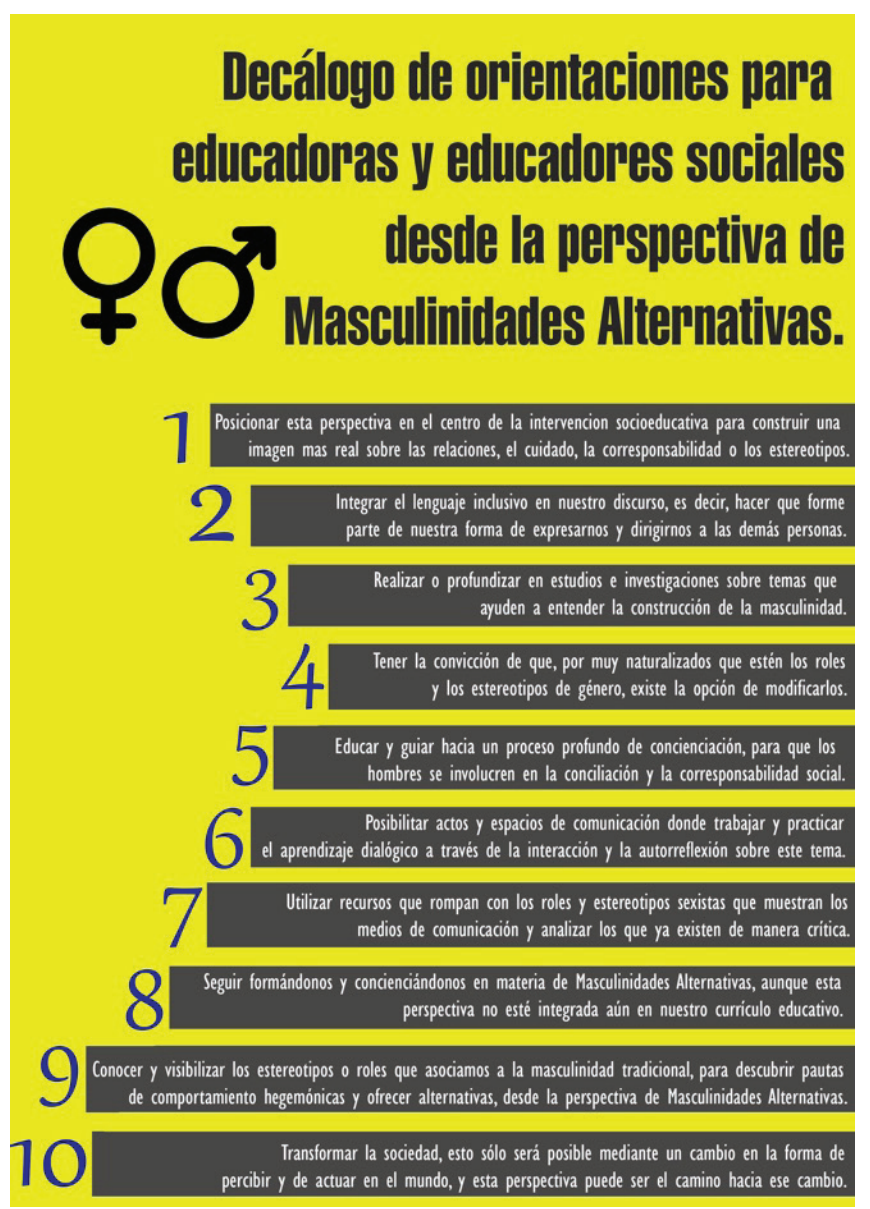




\section{Referencias}

Aguayo, F. y Kimelman, E. (2016). Programa P Bolivia: un manual para la paternidad activa. Padres $y$ madres por una crianza positiva, compartida y sin violencia. Banco Interamericano de Desarrollo.

AHIGE. Asociación de Hombres por la Igualdad de Género (25 de junio de 2020). Los hombres ganamos con la igualdad. https://cutt.ly/rjzWzNb

Amorós, C. (2006). La gran diferencia y sus pequeñas consecuencias para las luchas de las mujeres. Cátedra.

Aresti, N. (2020). La historia de las masculinidades, la otra cara de la historia de género. Ayer, (117), 333-347.

Azpiazu, J. (2017). Masculinidades y feminismo. Virus Editorial.

Banco Mundial (25 de junio de 2020). Crecimiento de la población (\% anual). https://cutt.ly/1jzWn5s

Bas, E. (2015). Educación Social y Género. En: J. J. Leiva, V. M. Martín, E. S. Vila y J. E. Sierra (Coords.), Género, educación y convivencia (pp. 113-124). Dykinson.

Belloch, C. (25 de junio de 2020). Las Tecnologias de la Información y Comunicación en el aprendizaje. Universitat de Valencia, Entornos Virtuales de Formación. https://cutt.ly/DjzWAbn

Bergara, A., Rivière J. y Bacete R. (Eds.). (2008). Los hombres, la igualdad y las nuevas masculinidades. Emakunde.

Bonino, L. (2000). Varones, género y salud mental: deconstruyendo la «normalidad» masculina en A. Carabí, y M. Segarra, (Eds.). Nuevas masculinidades (pp. 41-64). Icaria.

Bonino, L. (2002). Masculinidad hegemónica e identidad masculina. Dossiers Feministes, (6), 7-35. https://cutt.ly/8jzW1bV

Bonino, L. (2003). Los hombres y la igualdad con las mujeres en C. Lomas (Ed.), ¿Todos los hombres son iguales? (pp. 105-144). Paidós.

Boscán, A. (2008). Las nuevas masculinidades positivas. Utopia y Praxis Latinoamericana, 13(47), 93-106.
Bourdieu, P. (2000). La dominación masculina. Anagrama.

Carabí, A. (2000). Construyendo nuevas masculinidades: una introducción en A. Carabí, y $\mathrm{M}$. Segarra (Eds.), Nuevas masculinidades (pp. 15-28). Icaria.

Cardeñosa, P. (2019). Perspectiva feminista en la intervención socioeducativa. Boletín Gizaberri, (77), 4-6. Colegio de Educadores y Educadoras del País Vasco. https://cutt.ly/ojzW3g6

Castro, O. y Spoturno, M. L. (2020). Feminismos y traducción: apuntes conceptuales y metodológicos para una traductología feminista transnacional. Mutatis Mutandis: Revista Latinoamericana De Traducción, 13(1), 11-44.

Connell, R. W. (1987). Gender and power: Society, the person and sexual politics. Stanford University Press.

De Beauvoir, S. (1981). El segundo sexo. Siglo XX.

Duque, E. (Coord.). (2015). IDEALOVEyNAM. Socialización preventiva de la violencia de género. CNIIE. Ministerio De Educación, Cultura y Deporte.

Emakunde. (2018). CIFRAS 2018-Mujeres y Hombres en Euskadi (Informe). Eusko Jaurlaritza. https:// cutt.ly/MjzW7n9

Espada, J. M. (7 de mayo de 2004). Poder, masculinidad y virilidad (ponencia). Curso Técnico Especialista en Igualdad de Oportunidades en el Empleo, IMUMEL, Albacete. https://cutt.ly/ kjzW6tM

Facio, A. y Fries, L. (1999). Feminismo, género y patriarcado en A. Facio y L. Fries (Eds.), Género y Derecho. La Morada.

Flecha, R., Puigvert, L. y Ríos, O. (2013). The New Alternative Masculinities and the overcoming of gender violence. International and Multidisciplinary Journal of Social Sciences, 2(1), 88-113. http://dx.doi,org/10.4471/rimcis.2013.14

Fontenla, M. (25 de junio de 2020). ¿Qué es el patriarcado? Mujeres en Red. El periódico Feminista. https://cutt.ly/kjzEifn

Freedman, J. (2004). Feminismo. ¿Unidad o conflicto? Narcea. 
Freire, P. (1978). La educación como práctica de la libertad. Siglo XXI.

Freire, P. (1997). Pedagogía de la autonomía. Siglo XX. González, M. (2019). Procesos de participación de mujeres y hombres y creación de redes para el impulso de la igualdad. Grupo Editorial Ra-Ma.

Hardy, E. y Jiménez, A. L. (2001). Masculinidad y Género. Revista Cubana Salud Pública. 27(2) 77-88.

Ibarra. P. (2005). Manual de sociedad civil y movimientos sociales. Editorial Síntesis.

Jociles, M. I. (2001). El estudio sobre las masculinidades. Panorámica general. Gazeta de Antropología. (17). https://cutt.ly/ujzEsR0

Kaufman M. (1994). Men, feminism and mens contradictory experiences of power en $\mathrm{H}$. Broad $\mathrm{y}$ M. Kaufman (Eds.), Theorizing masculinities (pp. 142-164). Sage. https://cutt.ly/pjzExqW

Kimmel, M. (1997). Homofobia, temor, vergüenza y silencio en la identidad masculina en T. Valdés y J. Olavarría (Eds.), Masculinidadles. Poder y crisis, (pp. 49-63). Ediciones de las Mujeres.

Kimmel, M. (2001). Masculinidades globales: restauración y resistencia en C. Sánchez-Palencia, y J. C. Hidalgo (Eds.), Masculino plural: Construcciones de la masculinidad (pp. 47-76). Universidad de Lleida.

Leiva, J. J., Martín, V. M., Vila, E. S. y Sierra, J. E. (Coords.). (2015). Género, educación y convivencia. Dykinson.

Lerner, G. (1990). La creación del patriarcado. Crítica. Llano, L., Mier, P. y Río, A. M. (2020). Introducir la perspectiva de género a través del juego. Educación para el bien común: Hacia una práctica critica, inclusiva y comprometida socialmente (pp. 851-863). Octaedro.

Lorente, M. (2009). Los nuevos hombres nuevos. Los miedos de siempre en tiempos de igualdad. Ediciones Destino.

Martín, V. M. y Vila, E. S. (2015). Igualdad de género y pedagogía/educación social: un encuentro ontológico en J. J. Leiva, V. M. Martín, E. S. Vila, y J. E. Sierra (Coords.), Género, educación y convivencia (pp. 141-149). Dykinson.
Marugán, B. (2020). Género. Eunomia: Revista En Cultura De La Legalidad, (18), 199-213. https:// doi.org/10.20318/eunomia.2020.5273

Medina-Vicent, M. (2020). Los retos de los feminismos en el mundo neoliberal. Revista Estudios Feministas, 28(1), 1-12. https://doi.org/ 10.1590/1806-9584-2020v28n157212

Millet, K. (1995). Politica sexual. Cátedra.

Ministerio de Asuntos Exteriores de Cooperación (2018). Consejo de Europa. Estrategia de Igualdad de Género 2018-2023. https://cutt.ly/njzEWaF

Otegui, R. (1999). La construcción social de las masculinidades. Política y Sociedad, (32), 151-160.

Pantoja, L., Alonso, M. J., Arandia, M., Remiro, A., Rodríguez, I. y Rubio, D. (2018). El código deontológico de la educación social: una visión desde la práctica profesional. Ediciones Beta III Milenio.

Peña, J. C. y Ríos, O. (2011, 7 de octubre). Actos comunicativos que promueven nuevas masculinidades en los centros educativos (comunicación). Congreso Iberoamericano de Masculinidades y Equidad: Investigación y Activismo-CIME, Barcelona.

Portell, D. y Pulido, C. (2012). Communicative acts which promote new masculinities. Overcoming hegemonic masculinity in the workplace and the school. Masculinities and Social Change, 1(1), 61-80.

Radkau, V. (1986). Hacia una historiografía de la mujer. Nueva Antropología, 3(30), 77-94.

Reverter, S. y Medina-Vicent, M. (2020). El feminismo en 35 hashtags. Catarata.

Ríos, O. (2015). Nuevas masculinidades y educación liberadora. Intangible Capital, 11(3), 485-507. http://dx.doi.org/10.3926/ic.654

Rodríguez, A. (2017). Masculinidades en tertulia: un estudio de las posibilidades del diálogo y de la interacción para la transformación hacia la equidady la igualdad de género. [Tesis doctoral, Universidad de Barcelona]. https://cutt.ly/SjzEOhM

Soler, M. y Flecha, R. (2010). Desde los actos de habla de Austin a los actos comunicativos: Perspectivas desde Searle, Habermas y CREA. Revista Signos, (43), 363-375. 
Soto, G. (2013). Nuevas masculinidades o nuevos hombres nuevos: el deber de los hombres en la lucha contra la violencia de género. Scientia Helmántica. Revista Internacional de Filosofía, (1), 95-106.

Torras-Gómez, E., Puigvert, L., Aiello, E. y Khalfaoui, A. (2020). Our right to the pleasure of falling in love. Frontiers in Psychology, 10(3068), 1-11.

Varela, N. (2020). El tsunami feminista. Nueva Sociedad, (286), 93-106.
Vila, E. S. y Martín, V. M. (2015). ¿Qué parte de mí dejo fuera? identidad de género, nuevas masculinidades y educación en J. J. Leiva, V. M. Martín, E. S. Vila, y J. E. Sierra (Coords.). Género, educación y convivencia (pp. 161-170). Dykinson.

Ytarte, R. M. (2015). Género y educación social: formación académica y competencias profesionales en J. J. Leiva, V. M. Martín, E. S. Vila, y J. E. Sierra (Coords.). Género, educación y convivencia (pp. 151-165). Dykinson. 